\title{
Alan F. Schatzberg, MD, on the state of psychiatry
}

Awais Aftab, MD, and Alan F. Schatzberg, MD

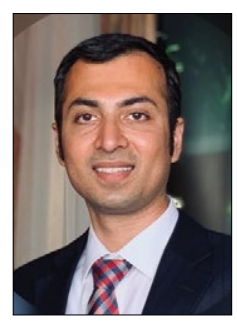

Awais Aftab, MD

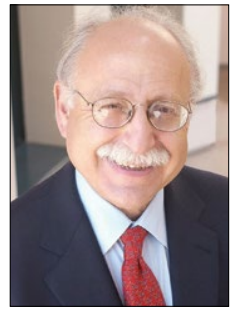

Alan F. Schatzberg, MD

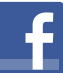

Discuss this article at www.facebook.com/ MDedgePsychiatry

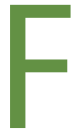
or this Psychiatry Leaders' Perspectives, Awais Aftab, MD, interviewed Alan F. Schatzberg, MD. Dr. Schatzberg is the Kenneth T. Norris, Jr., Professor of Psychiatry and Behavioral Sciences at Stanford University. He served as the Chair of the Department at Stanford until 2010 and currently directs the Stanford Mood Disorders Center. He was the 136th president of the American Psychiatric Association (APA) (2009-2010). He has been an active investigator in the biology and psychopharmacology of depressive disorders, and has authored more than 700 publications and abstracts, including Schatzberg's Manual of Clinical Psychopharmacology. Dr. Schatzberg is also the coeditor of the Textbook of Psychopharmacology with Charles B. Nemeroff, MD, PhD. He is a Past President of the American College of Neuropsychopharmacology (ACNP) and the Society of Biological Psychiatry, and was also the Secretary-General of the International Society of Psychoneuroendocrinology (ISPNE). In 2003, he was elected to the Institute of Medicine of the National Academy of Sciences (National Academy of Medicine). He has received numerous prestigious awards, including the 2005 Distinguished Service in Psychiatry Award from the American College of Psychiatrists, the 2005 Falcone Award from the National Alliance for Research in Schizophrenia and Affective Disorders, the 2014 Kraepelin Gold Medal from the Max Planck Institute of Psychiatry, the 2015 Gold Medal from the Society of Biological Psychiatry, the 2015 Lifetime Achievement Award of the
ISPNE, the 2017 Julius Axelrod Mentorship Award from the ACNP, the 2018 Donald Klein, MD, Lifetime Achievement Award from the American Society of Clinical Psychopharmacology, and the 2018 Jules Marmor, MD, Award for Biopsychosocial Research from the APA.

Dr. Aftab: You have devoted much of your career to the development of psychopharmacology. What is your perspective on where the field of psychopharmacology stands at present, especially amid the widespread recognition of "treatment resistance" as a pervasive phenomenon and the scarcity of validated neurobiologic etiological models for psychiatric disorders?

Dr. Schatzberg: We have made considerable progress in the development of new classes of agents for major depression, but as we develop new agents, we still see a large percentage of patients who do not seem to demonstrate adequate responses, particularly in major depressive disorder. This has driven us to look for agents that work differently than previous ones. Although we have some new agents with

Dr. Aftab is a Clinical Assistant Professor, Psychiatry, Case Western Reserve University, Cleveland, Ohio. Dr. Schatzberg is the Kenneth T. Norris, Jr., Professor of Psychiatry and Behavioral Sciences, and Director, Stanford Mood Disorders Center, Stanford University, Stanford, California.

\section{Disclosures}

Dr. Aftab reports no financial relationships with any companies whose products are mentioned in this article, or with manufacturers of competing products. Dr. Schatzberg has served as a consultant to Alto, ANeuroTech, Axsome, Boehringer Ingelheim , Compass, Delpor, Douglas, Janssen, Jazz, NeuraWell, Neuronetics, and Sage. He has equity in Alto, Corcept, Delpor, Epiodyne, NeuraWell, and Owl Insights. doi: 10.12788/cp.0202 
seeming efficacy and newer mechanisms of action, eg, esketamine, these have largely been derived from clinical, often serendipitous, observations of antidepressant effects rather than from prospective development based on a known pharmacological effect or a biological construct of the disorder. Another intriguing and possibly effective anxiolytic and antidepressive agent is psilocybin, whose potential use is largely derived from clinicians who found it helpful in their practices in combination with psychotherapy. These $2 \mathrm{dem}-$ onstrate how as we branch out into new territory, we find ourselves moving more and more toward drugs of known clinical risk; eg, mind-altering agents or drugs of abuse. These agents may offer riskbenefit ratios that can ultimately prove to be less attractive than what we might have wanted when we ventured on the journey. Unfortunately, there has been little dialogue about the limitations of several of these agents.

In the case of esketamine, the notion has been that the drug is a blocker of the $\mathrm{N}$-methyl-D-aspartate (NMDA) glutamate receptor, suggesting it is relatively safe. However, we and others have now clearly demonstrated that the antidepressant effect in humans and the antidepressant behavioral effects in rodents are mediated via the mu opioid receptor. ${ }^{1-3}$ This implies an abuse and dependence risk, and it is concerning that there has been much emphasis on ketamine's effect on the NMDA glutamate receptor and not enough on this opioid effect, even though the very active enantiomer esketamine demonstrates relatively equal micromolar binding to both mu and NMDA receptors. Understanding the biology of major depression better and elucidating the key mechanisms of action of agents are both needed if we are to develop more effective and still safe agents.

One approach that has been applied recently is target validation that purports to use functional MRI to assess behavioral and cognitive effects of drugs to allow inferences regarding efficacy in specific disorders. As we have discussed in a recent paper published in the American Journal of Psychiatry, this can be quite misleading and may provide both false positive and negative information. From my perspective, these tests do not appear sensitive enough to screen for patients having a disorder, nor for assessing possible drug effects in those patients. Thus, it is unclear if they can provide answers today that we can be confident in.

Dr. Aftab: What do you see as some of the strengths of psychiatry as a profession?

Dr. Schatzberg: Psychiatry as a specialty combines 2 major perspectives-psychological processes and psychobiology-to develop methods for treating patients who suffer from disorders of the mind/brain. It is the most challenging of our specialties because we cannot study the brain directly. We cannot do procedures as we do in cardiology and pulmonology because they may prove dangerously invasive. That handsoff approach limits us, but for the curious it provides an opportunity to begin to unravel the processes that underlie brain functioning. Fortunately, we have therapies-both psychosocial and somatic - that can provide great relief to patients. These can be shown to be effective in sufficient numbers of patients to help many.

Dr. Aftab: Are there ways in which the status quo in psychiatry falls short of the ideal? What are our areas of relative weakness?

Dr. Schatzberg: We need to train our residents in a host of approaches, and not just medications and psychotherapy. They need to understand the basis of brain stimulation approaches (such as repetitive transcranial magnetic stimulation) as well as know how to apply them. We need to train residents

\section{Clinical Point}

As we branch out into new territory, we find ourselves moving more and more toward drugs of known clinical risk 


\section{Clinical Point}

Our classification
approaches ... need
to become more
rapidly adaptive to
research in the field

more in substance abuse problems and the biology of addiction if they are to better understand the risks of certain new classes of medication. Lastly, we need to train residents in the application of genomics, proteomics, and brain imaging to somatic treatment development.

Dr. Aftab: What is your perception of the threats that psychiatry faces or is likely to face in the future?

Dr. Schatzberg: The biggest threats come from ourselves. We need to do better with our classification approaches, such as the Diagnostic and Statistical Manual of Mental Disorders or the Research Domain Criteria. They need to become more rapidly adaptive to research in the field. We need to be more open to looking at what is a potentially dangerous trend in developing drugs of abuse and mindaltering drugs as therapeutics. We need to be able to demonstrate that telepsychiatry can be as effective as face-to-face treatment and should be reimbursed. Lastly, we need to develop better models for taking care of the psychiatric patient. We have too many patients and not enough psychiatrists.
Dr. Aftab: What do you envision for the future of psychiatry? What sort of opportunities lie ahead for us?

Dr. Schatzberg: I see the future as bright. Over the past 10 years, led by efforts at the APA, some while I was President, reimbursement has increased dramatically. Over the past 10 years, we have done well developing some new drugs and somatic therapies, and these will continue. Less than a decade ago, large pharmaceutical had abandoned psychiatric drug development and investment into biotech start-ups had waned to near zero. However, the last year few years have seen a dramatic surge in investment, and these should yield novel agents and ones that may be combined with innovative biomarkers as companions.

\section{References}

1. Williams NR, Heifets BD, Blasey C, et al. Attenuation of antidepressant effects of ketamine by opioid receptor antagonism. Am J Psychiatry. 2018;175(12):1205-1215. doi:10.1176/appi.ajp.2018.18020138

2. Williams NR, Heifets BD, Bentzley BS, et al. Attenuation of antidepressant and antisuicidal effects of ketamine by opioid receptor antagonism. Mol Psychiatry. 2019;24(12):1779-1786. doi:10.1038/s41380-019-0503-4

3. Bonaventura J, Lam S, Carlton M, et al. Pharmacological and behavioral divergence of ketamine enantiomers: implications for abuse liability. Mol Psychiatry. 2021;10.1038/ s41380-021-01093-2. doi:10.1038/s41380-021-01093-2

4. Schatzberg AF. Can target engagement studies miss their targets and mislead drug development? Am J Psychiatry. 2021;178(5):372-374. doi:10.1176/appi.ajp.2020.21030247 